\title{
Energizer keep going: 100 years of superconductivity
}

It has been 100 years since Heike Kamerlingh Onnes discovered superconductivity on April 8, 1911. Amazingly, this field is still very active and keeps booming, like a magic. A lot of new phenomena and materials have been found, and superconductors have been used in many different fields to improve our lives. Onnes won the Nobel Prize for this incredible discovery in 1913 and used the word superconductivity for the first time. Onnes believed that quantum mechanics would explain the effect, but he could not produce a theory at that time. Now we know superconductivity is a macroscopic quantum phenomenon. An important breakthrough is the development of Bardeen-Cooper-Schrieffer (BCS) theory in 1957 which gives a microscopic mechanism to the origin of superconductivity. The key idea is the formation of Cooper pairs - condensation of electrons into Bosons. In metals and many conventional superconductors, the electron-phonon coupling is the driving force for the formation of Cooper pairs. In 1986, a new family of superconductors with high-transition temperature (high- $T_{\mathrm{c}}$ ) based on copper-oxides was discovered by Johannes Bednorz and Karl Müller, opening a new era of superconductivity. The traditional pairing mechanism does not work for these materials, and we call them unconventional superconductors. So far, the formation of Cooper pairs is believed mainly from the spin fluctuations, i.e., electron-magnon coupling, although this in itself is an issue under debate. In 2008, another family of unconventional superconductors - iron-based superconductors was discovered. It immediately caused great excitement in the community because this is only the second class of high- $T_{\mathrm{c}}$ superconductors in addition to the copper oxides. Intensive studies have shown that the origin of superconductivity in the iron-based superconductors may be similar to the cuprate superconductors, where magnetism plays an important role in the formation of Cooper pairs. One distinct feature of the iron-based superconductors is its multiorbital nature, which makes it special in studying both localized and itinerant electrons. Some other superconductors like heavy femion materials, organic superconductors also show unconventional properties.

This year marks the 100 anniversary of the discovery of superconductivity. The journal Frontiers of Physics decided to take this opportunity to organize a high-quality special issue which is dedicated to the diversified fields and important frontiers on superconductivity. This group of articles include work by Beekman and Zaanen on electrodynamics of Abrikosov vortices, a reflection on the contrast between the Cooper pairing in iron-based and conventional superconductors by Wang and Lee, a News \& Views on iron-based superconductors by Yao, a Perspective by Mandrus on how serendipity plays an important role in discovering new superconductors, a review article by Weng on the relevance of Mott physics to high- $T_{\mathrm{c}}$ superconductivity, a summary of the multiorbital Hubbard Models for the iron-based superconductors by Dagotto and co-workers, a summary of recent small angle neutron scattering work on vortex lattices in type-II superconductors by Eskilden, a short review by Li and Dai on spin fluctuations in various unconventional superconductors, a summary on how to use angle resolved photoemission data to deduce the microscopic electron pairing mechanism by Choi, Varma, and Zhou, a brief review on structural, magnetic and electronic properties of a newly-discovered iron-chalcogenide $A_{x} \mathrm{Fe}_{2-y} \mathrm{Se}_{2}$ superconductor by Zhou and co-workers, a review on nuclear magnetic resonance studies of vortices in high temperature superconductors, and finally, a brief review on the recent studies of the upper critical field and its anisotropy in a few typical series of the iron-based superconductors by Yuan and co-workers.
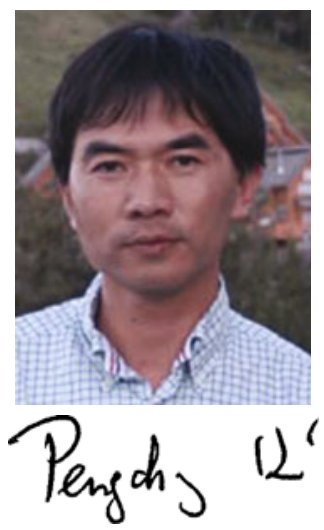

Professor Pengcheng Dai

Department of Physics and Astronomy,

The University of Tennessee, Knoxville, USA, and National Laboratory for Superconductivity,

Institute of Physics, CAS, Beijing, China E-mail:pdai@utk.edu
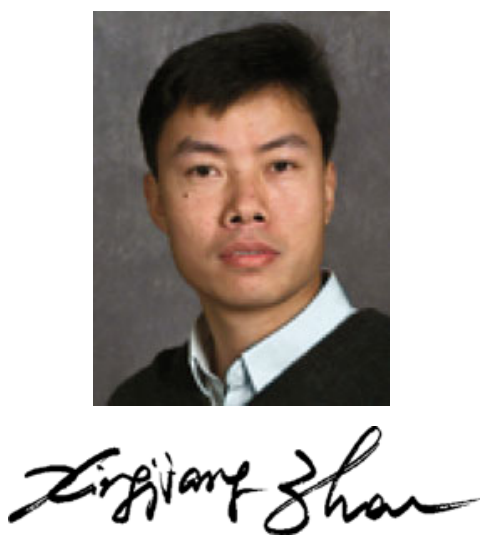

Professor Xing-jiang Zhou National Laboratory for Superconductivity, Institute of Physics, CAS, Beijing, China E-mail: XJZhou@aphy.iphy.ac.cn
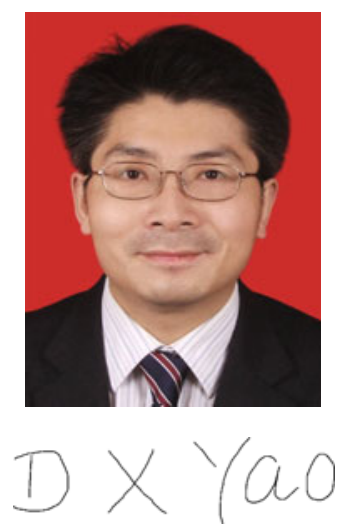

Professor Dao-xin Yao School of Physics and Engineering, Sun Yat-Sen University, Guangzhou, China E-mail: yaodaox@mail.sysu.edu.cn 